\title{
Probiotics modulate gut microbiota and improve insulin sensitivity in DIO mice
}

\author{
Renata A. Bagarolli ${ }^{a}$, Natália Tobar ${ }^{\mathrm{a}}$, Alexandre G. Oliveira ${ }^{\mathrm{b}}$, Tiago G. Araújo ${ }^{\mathrm{a}}$, Bruno M. Carvalho ${ }^{\mathrm{c}}$, \\ Guilherme Z. Rocha ${ }^{a}$, Juliana F. Vecina ${ }^{a}$, Kelly Calisto ${ }^{a}$, Dioze Guadagnini ${ }^{a}$, Patrícia O. Prada ${ }^{a}$, Andrey Santos ${ }^{a}$, \\ Sara T.O. Saad ${ }^{a}$, Mario J.A. Saad ${ }^{\mathrm{a}, *}$ \\ ${ }^{a}$ Department of Internal Medicine, State University of Campinas, 13081-970, Campinas, SP, Brazil \\ ${ }^{\mathrm{b}}$ Department of Physical Education, São Paulo State University (UNESP), Bioscience Institute, Rio Claro, SP, Brazil \\ ${ }^{\mathrm{C}}$ Department of Biology Science, Federal University of Pernambuco, Recife, PE, Brazil
}

Received 9 March 2017; received in revised form 5 July 2017; accepted 17 August 2017

\begin{abstract}
Obesity and type 2 diabetes are characterized by subclinical inflammatory process. Changes in composition or modulation of the gut microbiota may play an important role in the obesity-associated inflammatory process. In the current study, we evaluated the effects of probiotics (Lactobacillus rhamnosus, L. acidophilus and Bifidobacterium bifidumi) on gut microbiota, changes in permeability, and insulin sensitivity and signaling in high-fat diet and control animals. More importantly, we investigated the effects of these gut modulations on hypothalamic control of food intake, and insulin and leptin signaling. Swiss mice were submitted to a high-fat diet (HFD) with probiotics or pair-feeding for 5 weeks. Metagenome analyses were performed on DNA samples from mouse feces. Blood was drawn to determine levels of glucose, insulin, LPS, cytokines and GLP-1. Liver, muscle, ileum and hypothalamus tissue proteins were analyzed by Western blotting and real-time polymerase chain reaction. In addition, liver and adipose tissues were analyzed using histology and immunohistochemistry. The HFD induced huge alterations in gut microbiota accompanied by increased intestinal permeability, LPS translocation and systemic low-grade inflammation, resulting in decreased glucose tolerance and hyperphagic behavior. All these obesity-related features were reversed by changes in the gut microbiota profile induced by probiotics. Probiotics also induced an improvement in hypothalamic insulin and leptin resistance. Our data demonstrate that the intestinal microbiome is a key modulator of inflammatory and metabolic pathways in both peripheral and central tissues. These findings shed light on probiotics as an important tool to prevent and treat patients with obesity and insulin resistance.
\end{abstract}

(c) 2017 Elsevier Inc. All rights reserved.

Keywords: Probiotics; Gut microbiota; Insulin sensitivity

\section{Introduction}

Obesity, insulin resistance and type 2 diabetes (T2D) are metabolic disorders resulting from a combination of genetic and environmental factors including caloric intake and sedentary lifestyle [1,2]. In the past 10 years, data coming from different sources showed an important role of gut microbiota in the pathogenesis of obesity and type 2 diabetes [3-10].

Approximately $10^{13}$ and $10^{14}$ microorganisms live in a symbiotic manner in the human gastrointestinal tract. This relationship is essential for host physiology and metabolic health [11-13]. In this regard, the intestinal bacteria help in the host nutrients absorption and in the integrity of intestinal immune barrier $[13,14]$ and regulate host fat storage genes, thus regulating host energy homeostasis [15]. It is known that high-fat diet (HFD) can cause imbalances in the gut microbiota composition and in the intestinal epithelial layer integrity and that germ-free animals are protected from this deleterious effect of HFD [16].

\footnotetext{
* Corresponding author at: Departamento de Clínica Médica, FCM-UNICAMP, Cidade Universitária Zeferino Vaz, Campinas, SP, Brazil, 13081-970. Fax: +55 19 35218950.

E-mail address: msaad@fcm.unicamp.br (M.J.A. Saad).
}

Numerous strategies have been proposed to regulate these imbalances in gut microbiota composition (also known as intestinal dysbiosis) in obesity, such as the use of antibiotics, prebiotics and probiotics [7,17-19]. The latter, whose definition is live microbial food supplements which help maintain the balance of intestinal microflora $[18,19]$, have shown in some studies to be effective in the treatment of insulin resistance in both rodents and humans; however, the deep mechanisms that lead to such benefits are poorly understood [4,18-20]. Indeed, multistrain probiotics appear to be efficient against a wide range of intestinal issues [21,22]. Despite the absence of strong evidence for metabolic disease, we hypothesized that probiotic mixture would be more suitable to test in an experimental model of obesity. Furthermore, it is known that some strains have important immune modulatory actions besides their effects on gut epithelial barrier function [23-25]. Actually, Lactobacillus rhamnosus is a special strain to treat a wide range of experimental liver diseases, with interesting metabolic modulations in this organ [26-28]. Thus, in the current study, we have used a mixture of probiotic content L. rhamnosus, L. acidophilus and Bifidobacterium bifidum to treat obese mice.

Emerging evidence has shown not only that gut microbiota is important for intestinal physiology but also that microbiota-gutbrain axis strongly influences the central nervous function and 
behavior [29-35]. Accordingly, it was previously hypothesized that energy balance regulation by central nervous system signaling is probably subject to an influence of gut microbiota [13]. Based on this hypothesis, we can also speculate that gut microbiota may also exert an influence in the control of food intake by hypothalamus, thus contributing to evolution of obesity. In the current study, we evaluated the effects of probiotics on gut microbiota, changes in permeability, and insulin sensitivity and signaling in HFD and control animals. More importantly, we investigated the effects of these gut modulations on hypothalamic control of food intake, and insulin and leptin signaling.

\section{Research design and methods}

\subsection{Materials}

Antibodies anti-JNK (SC1648), anti-phosphorylated JNK (SC6254), anti-IR $\beta$ (SC711), anti-phosphorylated IR $\beta$ (SC25103), anti-IRS1 (SC559), anti-phosphorylated IRS-1 (SC33956), anti-JAK2 (SC278), anti-MyD88 (SC11356), anti-NOD1 (SC 398696), anti- STAT3 (SC483), anti-phosphorylated STAT3 (SC8001), anti-occludin (SC5562) and antiZO-1 (SC10804) were purchased from Santa Cruz Biotechnology (Santa Cruz, CA, USA), except as follows: anti-Akt (\#9272), anti-phosphorylated Akt (\#9271), anti- $\alpha$-tubulin (\#2144), anti-phophorylated JAK2 (\#3776) and anti-TLR4 (\#2219), obtained from Cell Signaling Technology (Beverly, MA, USA). Routine reagents were purchased from SigmaAldrich (Saint Louis, MO, USA), unless otherwise specified.

\subsection{Animals}

UNICAMP Central Animal Breeding Center (Campinas, São Paulo, Brazil) provided 8-week-old male Swiss mice. Animals ( $n=6$ per group) were housed in individual cages with free access to water and rodent chow under a 12-h light/dark cycle. The mice composed randomly two groups: one group on chow diet ( $8 \%$ fat, $26 \%$ protein, $54 \%$ carbohydrate, as a percentage of total $\mathrm{kcal}$ ) (C group) and the second group on an HFD (55\% of energy derived from fat, $29 \%$ from carbohydrates and $16 \%$ from protein) (DIO group) for 12 consecutive weeks. After that, some mice in the DIO and $\mathrm{C}$ groups received daily a pool of probiotics for 5 weeks, and they composed the DIOPB and CPB groups, respectively. The Ethics Committee of the State University of Campinas approved all procedures.

\subsection{Probiotic administration protocol}

A pool of probiotics that included L. rhamnosus, L. acidophilus and B. bifidum (Futureceuticals, Momence, IL, USA) was given for 5 weeks daily $\left(6 \times 10^{8} \mathrm{CFU}\right.$ of each strain; final concentration of $1.8 \times 10^{9} \mathrm{CFU}$ of bacteria). Prior of gavage, the probiotics were diluted in $300 \mu \mathrm{l}$ of sterile water. DIO and C groups received only the vehicle (sterile water).

\subsection{Hormones and LPS determination}

Enzyme-linked immunosorbent assay kits were used to measure insulin (Millipore, St. Charles, MO, USA), TNF- $\alpha$, IL-6 and glucagon like peptide 1 (GLP-1) (Thermo Fisher Scientific, Rockford, IL, USA). LPS was quantified using a commercially available Limulus Amebocyte Assay (Cambrex, Walkersville, MD, USA), according to the manufacturer's protocol.

\subsection{Tissue extraction, immunoprecipitation and protein analysis by immunoblotting}

Overnight-fasted mice were anesthetized; the abdominal cavity was opened, the vena cava was exposed, and $\mathrm{NaCl}(0.9 \%$ wt./vol.) solution or insulin $\left(10^{-6} \mathrm{~mol} / \mathrm{l}\right)$ was injected. Liver, ileum, muscle and hypothalamus were removed, minced coarsely and homogenized in lysis buffer. Lysates were process as previously described [36].

\subsection{Real-time polymerase chain reaction (PCR)}

Total RNA was obtained from different tissues for all four groups of mice according to the methods published previously [37]. Quantitative PCR was performed in an ABI 5700 Sequence Detector System (Applied Biosystems) using SybrGreen PCR Master Mix (Applied Biosystems, Carlsbad, CA, USA) (primers described in Supplementary Table 1).

\subsection{Metagenome profile}

Fecal samples were frozen in liquid nitrogen and stored at $-80^{\circ} \mathrm{C}$ until use. At this time, DNA was extracted using the QIAamp DNA Stool Mini Kit (Qiagen, Hilden, Germany). DNA from each sample was used to amplify the V2-V3 regions of 16S rRNA genes. PCR amplification, pyrosequencing of the PCR amplicons and quality control of raw data were performed as described previously [38]. Samples were then sequenced in GS FLX Titanium (Roche Applied Science, Mannheim, Germany). The readouts obtained from the sequencing were analyzed by bioinformatics using basic local alignment search tool X (BLASTX), observed with METAREP software and compared according to phylum prevalence among groups.

\subsection{Liver and adipose tissue histology}

Liver and epididymal white adipose tissues were excised and processed as described previously [7]. Sections ( $5 \mu \mathrm{m}$ ) were obtained and stained by hematoxylin and eosin to assess morphology. Tissue analyses were performed blinded by an expert pathologist (A.A.S.). Adipose tissue was evaluated by crown-like structure (CLS) density (average CLS number within 10 high-power fields per animal) and mean adipocyte surface area, using Imagelab Analysis software, as previously described [7].

\subsection{Adipose tissue immunohistochemistry}

Tissue sections, $5 \mu \mathrm{m}$, were mounted on silanized glass slides, processed and incubated with an epidermal-growth-factor-like compound containing mucin-like hormone receptor-like 1 (F4/80) primary antibody, as previously described [39]. Antibody staining was performed using an IHC-peroxidase kit (Advance HRP, Dako CytoMation, Carpinteria, CA, USA) according to the manufacturer's instructions. Three different high-power fields from three different sections were evaluated blinded by an expert pathologist (A.A.S.). The total number of nuclei of F4/80-expressing cells was counted for each field, and the area occupied by these cells was measured.

\subsection{In vivo experiments}

Hyperinsulinemic-euglycemic clamp was performed in anesthetized overnight-fasted mice immediately after catheterization. Prime insulin (Human recombinant insulin, Eli Lilly, Indianapolis, IN, USA) was continuously infused ( $30 \mathrm{mU} \mathrm{kg}^{-1} \mathrm{~min}^{-1}$ ) up to $120 \mathrm{~min}$. Blood glucose was measured at 5-min intervals, and glucose (5\% wt./vol.) was infused at a variable rate to maintain blood glucose at fasting levels. Glucose tolerance test was performed in 8-h fasted mice as described before [36]. To determine insulin sensitivity in the hypothalamus, stereotaxic surgery was employed with 26-gauge stainless steel indwelling guide cannula aseptically placed into the third ventricle (coordinates of $-1.8 \mathrm{~mm}$ posterior to the bregma and $5.0 \mathrm{~mm}$ below the surface of the skull) in Swiss mice under anesthesia as previously described $[8,40]$. Fasted mice for $12 \mathrm{~h}$ received intracerebroventricular (ICV) infusion of vehicle or insulin $(200 \mathrm{mU}$ in $2 \mu \mathrm{l}$; Eli Lilly and Company, Indianapolis, IN, USA) at 8:00 a.m. Food 
intake was recorded over 4 and 12 h. Next day, same mice were fasted for $12 \mathrm{~h}$ and received ICV infusion of vehicle or insulin 15 min before the hypothalamic tissue dissection. To determine leptin sensitivity in the hypothalamus, after 5 weeks of probiotics treatment, five mice from each group received an intraperitoneal (IP) injection of recombinant leptin (3 mg/kg body weight; Calbiochem, San Diego, CA, USA) or saline at 6:00 p.m. on 5 consecutive days, as previously described [41]. Food intake were measured daily after the leptin injection. For hypothalamic analysis of the leptin signaling, immunoblotting was performed, and the leptin was IP injected $45 \mathrm{~min}$ before of the hypothalamic tissue dissection.

\subsection{Statistical analysis}

Data are expressed as means \pm S.E.M., and the number of independent experiments is indicated. The results of blots are presented as direct comparisons of bands or spots in autoradiographs and quantified by optical densitometry (Scion Image; Scion Corporation, Frederick, MD). For statistical analysis, the groups were compared using a two-way analysis of variance with the Bonferroni test for post hoc comparisons and $t$ test for comparison of only two groups. The level of significance adopted was $P<.05$.

\section{Results}

3.1. Probiotic reduces weight gain and fat pad improves glucose tolerance and insulin resistance in DIO mice

Probiotic-treated mice receiving an HFD gained significantly less weight (DIOPB $11.4 \pm 1.2 \mathrm{~g}$ vs. DIO $16.9 \pm 1.4 \mathrm{~g} ; P<.05$ ) during the 5 weeks of the experiment and had reduced food intake compared with animals that did not receive probiotics (DIOPB $2.9 \pm 0.2 \mathrm{~g} /$ day $v s$. DIO $3.4 \pm 0.3 \mathrm{~g} / \mathrm{da} ; P<.01$ ). In order to avoid this bias, we submitted the nontreated animals to pair feeding on an HFD, and this group was called diet-induced obesity mice per fed (DIOPF).
Fig. 1 shows comparative data regarding the controls (C), controls treated with probiotics for 5 weeks (CPB), DIOPF and DIO mice treated with probiotics for 5 weeks (DIOPB). As expected, the DIOPF and DIOPB mice showed a significant increase in both body and epididymal fat weight, along with higher levels of fasting glucose and insulin, when compared with animals fed on chow diet (Fig. 1A-D). However, the treatment with probiotics provided a marked decrease in weight gain (DIOPB $12.8 \pm 1.1 \mathrm{~g} v$ s. DIOPF $14.7 \pm 1.2 \mathrm{~g} ; P<.05$ ) and fat pad weight in 5 weeks and also in fasting blood glucose and serum insulin (Fig. 1A-D). Throughout the intraperitoneal glucose tolerance test (IPGTT), DIOPB mice showed improved glucose and insulin profiles compared to DIOPF animals (Fig. 1E and F). Likewise, the DIOPF group showed a striking reduction in the glucose infusion rate measured by the hyperinsulinemic-euglycemic clamp compared with control animals, and the probiotic treatment partially restored insulin sensitivity in DIOPF (Fig. 1G). It is important to mention that the probiotic treatment did not induce significant difference in any physiologic and metabolic parameters studied in animals fed on chow diet (Fig. 1A-G).

\subsection{Effects of probiotic treatment on insulin signaling in liver and muscle of DIO mice}

As expected, in liver and muscle of mice fed on HFD, insulinstimulated $\mathrm{Akt}^{\mathrm{Ser}} 473$ phosphorylation levels were significantly reduced compared to the control animals (Fig. 2A-B). On the other hand, the probiotic treatment completely recovered the insulininduced $\mathrm{Akt}^{\mathrm{Ser}} 473$ phosphorylation levels in both tissues when compared to the obese DIOPF (Fig. 2A-B). There was no difference in the Akt protein expression among $\mathrm{C}, \mathrm{CPB}$, DIOPF and DIOPB groups.

\subsection{Effects of probiotic treatment on the TLR4 signaling pathway in DIO mice}

The TLR4 protein levels in liver and muscle tissues were higher in the DIOPF animals compared to corresponding controls, though the
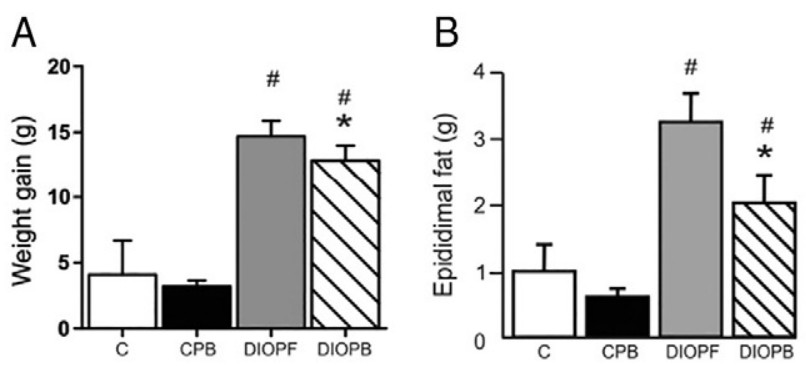

C
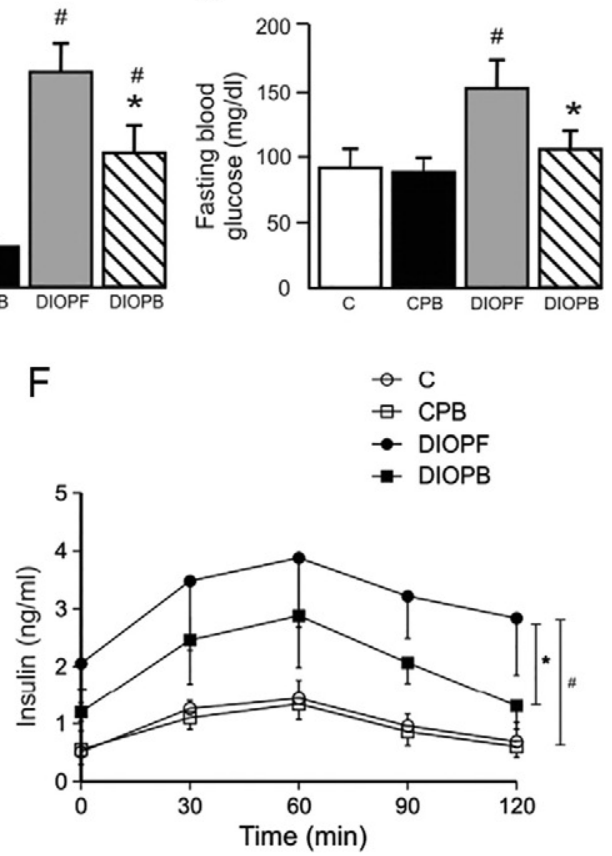

D

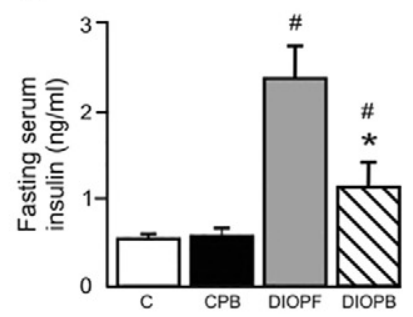

G

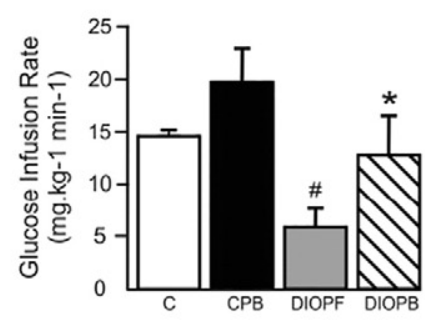

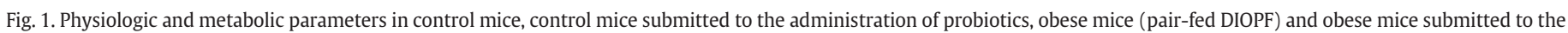

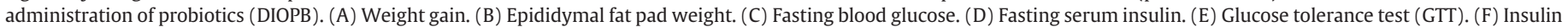
response curve during the GTT. $(G)$ Hyperinsulinemic-euglycemic clamp. Data are presented as means \pm S.E.M. of 6 to 10 mice per group. \#P<.05 vs. control, ${ }^{*} P<.05$ vs. DIOPF. 
A

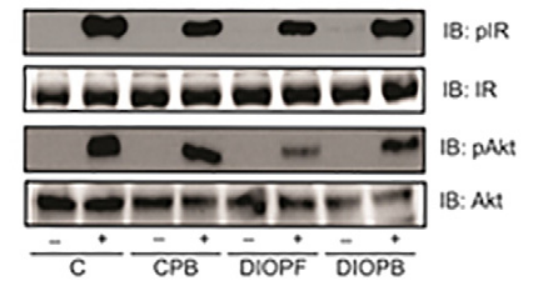

C



B

\section{MUSCLE}

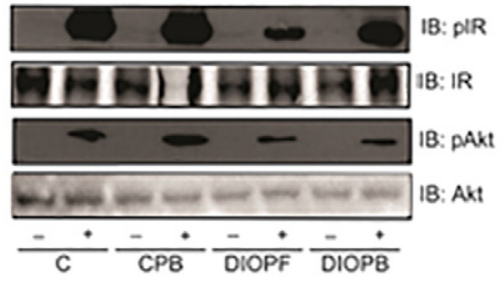

D

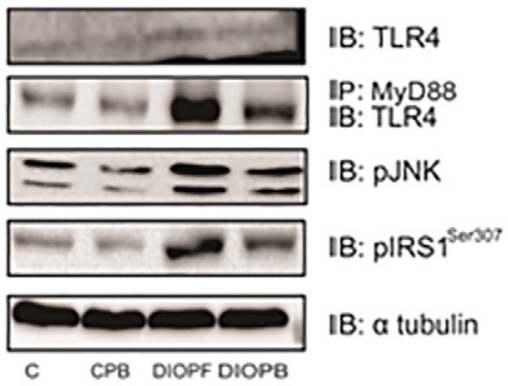

E
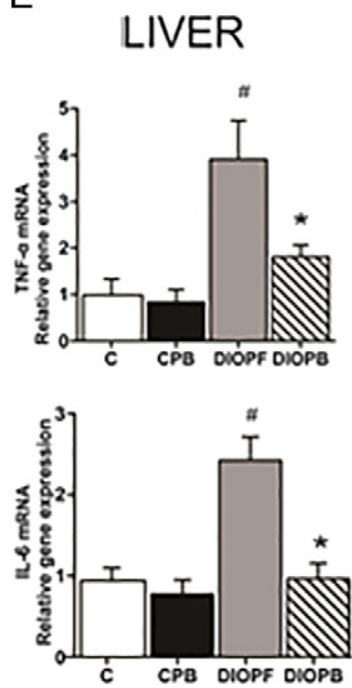

MUSCLE
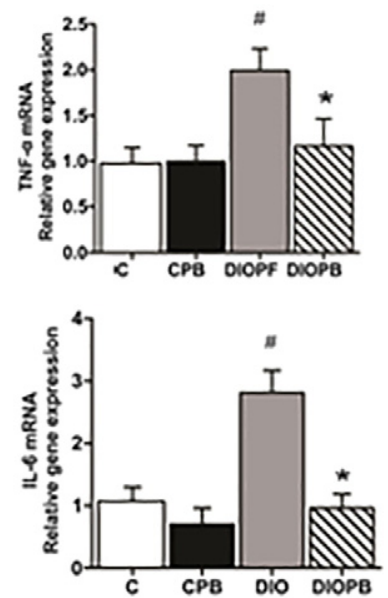

$\mathrm{F}$

\section{SERUM}
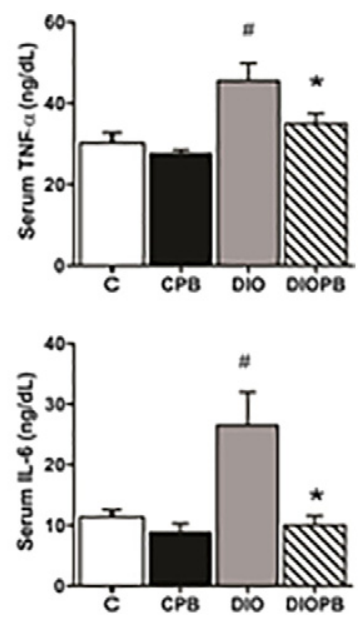

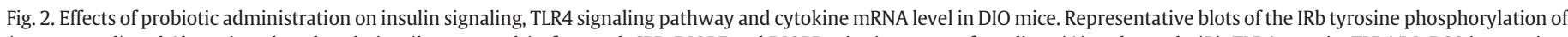

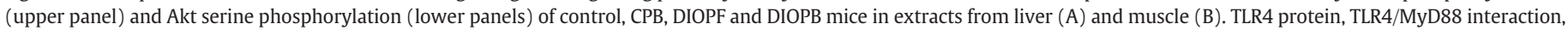

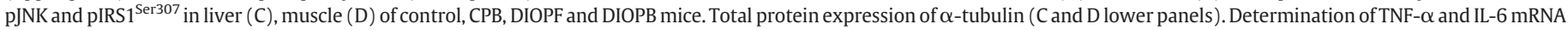

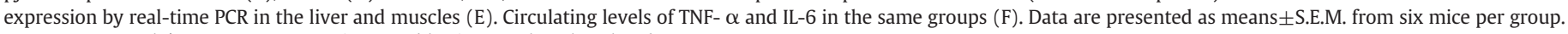
$\# P<.05$ vs. control, ${ }^{*} P<.05$ vs. DIOPF. IB, immunoblotting; p, phosphorylated.

use of PB was able to return TLR4 protein levels to baseline (Fig. 2C-D). As expected, the HFD activated the signaling of TLR4 in liver and muscle tissues of DIOPF animals as evidenced by the greater interaction of TLR4 with its adaptor protein MyD-88 along with increased activity of its downstream JNK (Fig. 2C-D). Such activation resulted in increased IRS- $1^{\text {Ser307 }}$ phosphorylation (Fig. 2C-D). By contrast, the administration of probiotics to obese animals was able to reduce TLR4 activation, downstream JNK phosphorylation and the subsequent IRS1 ${ }^{\mathrm{Ser} 307}$ phosphorylation (Fig. 2C-D). With regard to the control groups, the probiotic treatment did not provide changes on the TLR4 signaling (Fig. 2C-D).

\subsection{Probiotics reduce tissue cytokines $m R N A$ level in DIO mice}

To verify whether alterations in TLR4 signaling were reflected in increased proinflammatory cytokines, we examined TNF- $\alpha$ and IL-6 mRNA expression in the tissues of the studied groups, and these mRNA levels were higher in liver and muscle of the DIOPF as compared to the control group (Fig. 2E). In accordance with TLR4 signaling, the relative amounts of TNF- $\alpha$ and IL- 6 transcripts were markedly reduced in liver and muscle from DIOPB animals compared with DIOPF group (Fig. 2E). These benefits were also observed in circulating levels of TNF- $\alpha$ and IL6 (Fig. 2F). 


\subsection{Effects of probiotic treatment on gut microbiota of DIO mice}

The metagenomic sequencing analysis from feces of all groups showed profile changes among all studied groups. Among the control mice, treatment with probiotics increased the prevalence of Firmicutes and Actinobacteria, as well as decreased the occurrence of Bacteroidetes compared to untreated animals. In addition, by comparing control and DIO mice, regardless probiotic treatment, there were a greater increase in the prevalence of Bacteroidetes and a decrease in the Firmicutes and Actinobacteria phylum in the obese animals. Although this result disaccords with most studies in animals, we would like to emphasize that we previously observed similar result with Swiss mice [7]. Finally, the administration of probiotics in obese animals provided a continued presence of Bacteroidetes, an increase in the prevalence of Actinobacteria, along with a reduction in the phylum Firmicutes compared to DIOPF. The probiotics also promoted an increased variety of phyla presented in these animal feces (data not shown).

Fig. $3 \mathrm{~B}$ and $\mathrm{C}$ shows the distribution of some genera of Bacteroidetes and Firmicutes phyla in the DIOPF and DIOPB groups. Although probiotic treatment did not change the prevalence of Bacteroidetes in animals on a HFD, there was a major change in the concentration of certain genera, such as Bacteroides and Alistipes, populations of which increased in a significant way compared to levels in DIOPF (Fig. 3B).
A

C

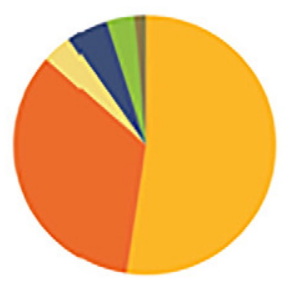

Bucteroidetes $\square$ Firmicutes
CPB

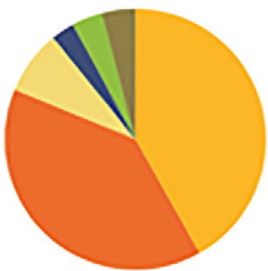

DIOPF

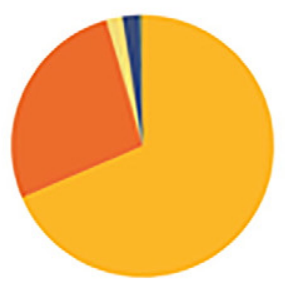

DIOPB

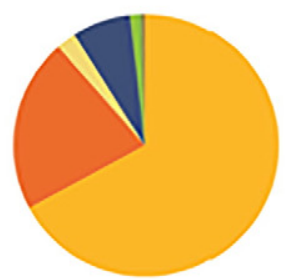

Cyanobactoria

$\square$ Protcobucteris

$\square$ Tenericutes

B

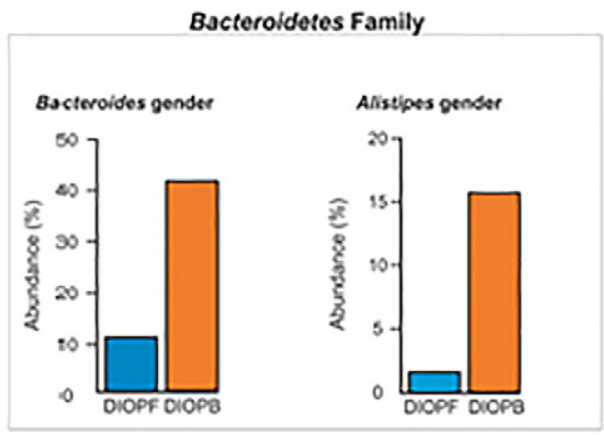

C

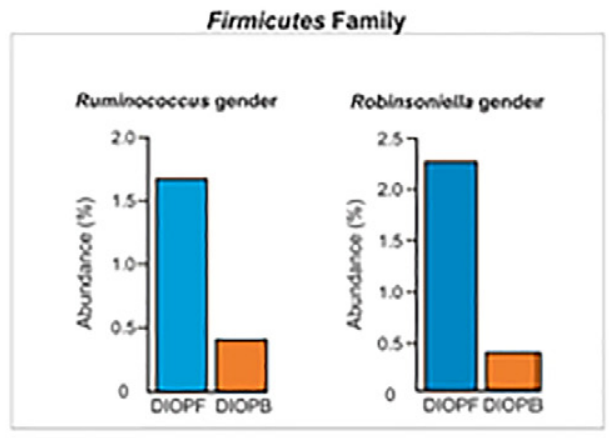

D

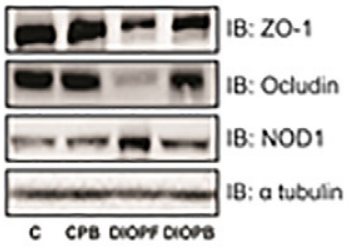

G

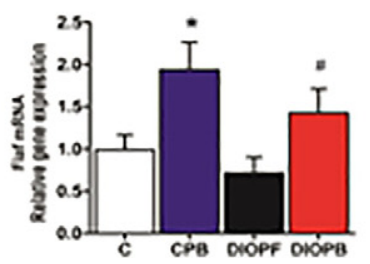

$E$

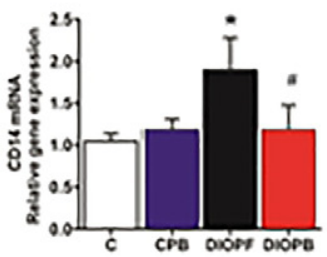

$\mathrm{H}$

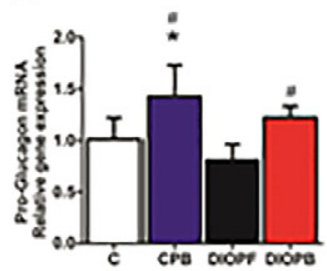

F

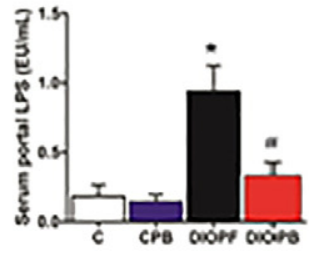

I

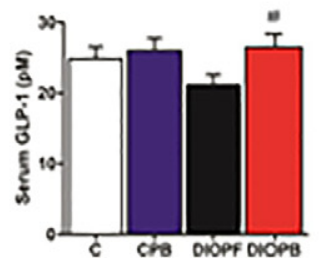

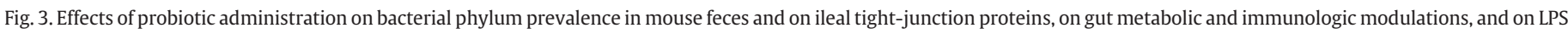

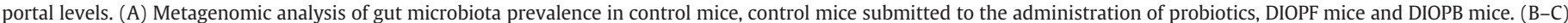

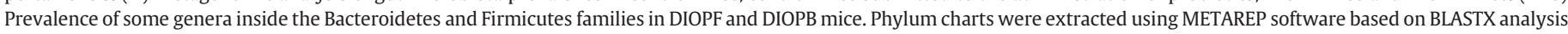

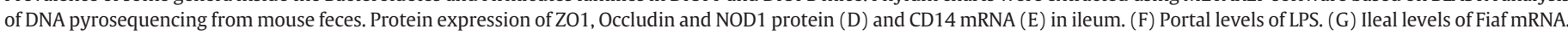

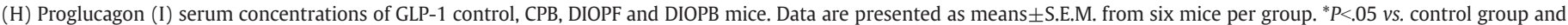
$\# P<.05$ vs. DIOPF. IB, immunoblotting. 
Previous data have shown that an increased taxonomic family Lachnospiraceae bacterium contributes to development of diabetes in obese mice [42-44]. Our data showed that Robinsoniella and Riminococcus genera from the Lachnospiraceae family were increased in DIOPF mice and showed reduction after PB treatment (Fig. 3C).

\subsection{Effects of probiotic treatment on gut metabolic and immunologic homeostasis}

We then examined the consequences that changes in gut microbiota provoked on tight-junction proteins and on important immune proteins situated in the ileum. ZO- 1 and Occludin protein and mRNA were reduced in DIOPF animals compared with controls, whereas the administration of probiotics restored partially these expressions (Fig. 3D). Furthermore, the main molecules involved in gut microbiota inflammation and bacterial translocation, NOD-1 and CD-14, had their expression increased in ileum of DIO animals, and the treatment with probiotics reversed this negative modulation (Fig. 3D and $\mathrm{E}$ ). As consequence of these changes, obese mice also showed increased levels of LPS in their portal circulation compared with the control mice, and this was prevented by probiotic treatment (Fig. 3F). Previous data showed that modulation of fasting-induced adipose factor (FIAF, a factor that increases triglycerides accumulation in adipose tissue) and GLP-1 might have a role in microbiota-induced insulin resistance/obesity [45]. Probiotic treatment in high-fat fed animals increased the expression of FIAF and proglucagon mRNAs in the ileum, as well as serum levels of GLP-1, compared with DIOPF (Fig. 3G-I). Probiotics had no such effect in control group (Fig. 3D-I).

\subsection{Effects of probiotic treatment on histology in liver and adipose tissue}

Histological sections from livers of DIOPF group showed the presence of innumerous fat vesicles compared to control sections, which characterizes hepatic steatosis. Nevertheless, treatment with probiotics prevented this fat accumulation in the liver parenchyma (Fig. 4A). Morphologic analysis of epididymal fat pad revealed significant differences on adipocyte size from DIO groups compared to control groups. However, between the DIO groups, we observed that the treatment with probiotics reduced the area of the adipocytes significantly but still far from the values of the controls animals. The HFD provided a huge macrophage infiltration in the adipose tissue, determined by the F4/80+ staining, but the administration of probiotics decreased such infiltration as well as the number of crown-like structures, which were abundant in DIOPF animals (Fig. 4B-D).
A

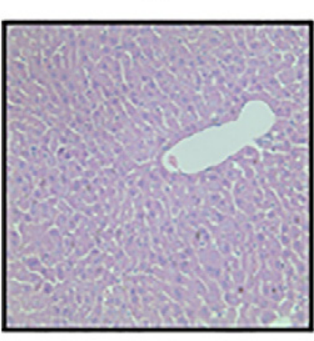

B
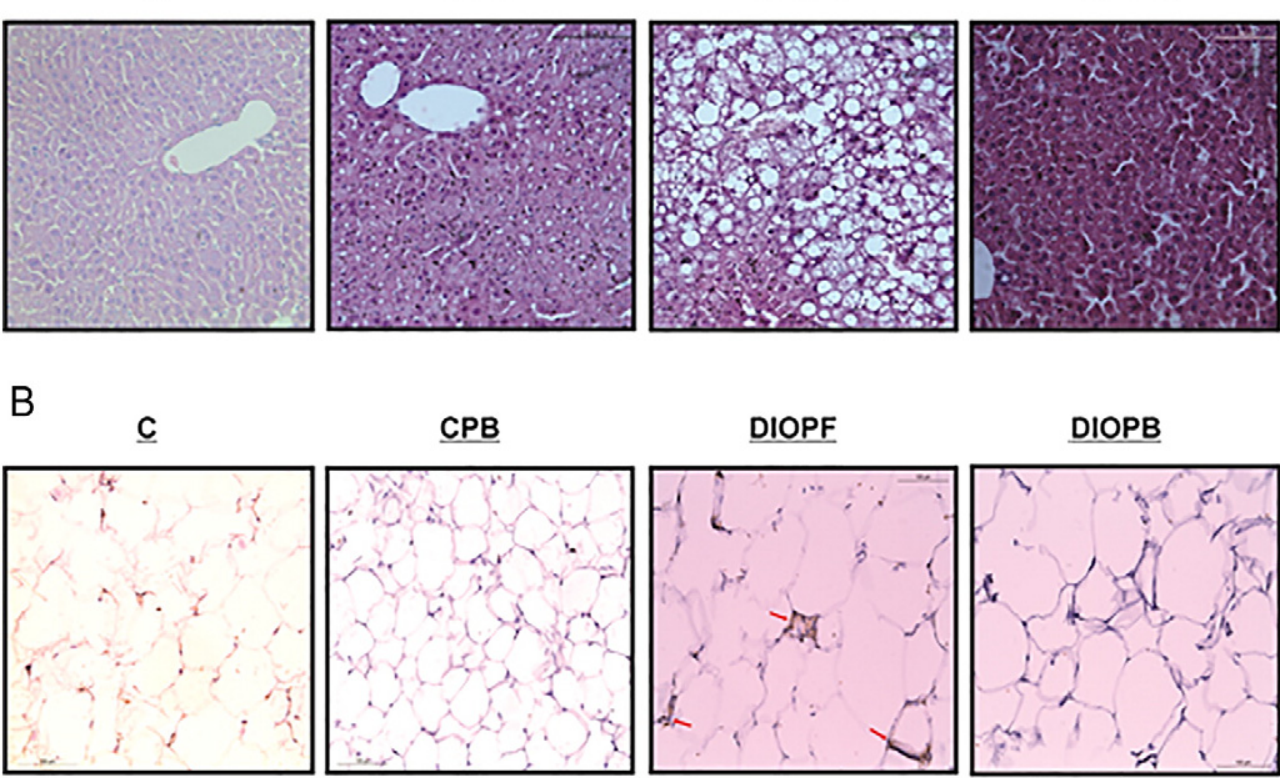

DIOPF
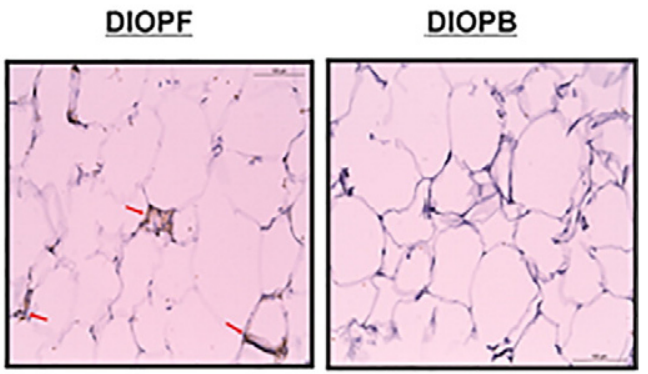

C

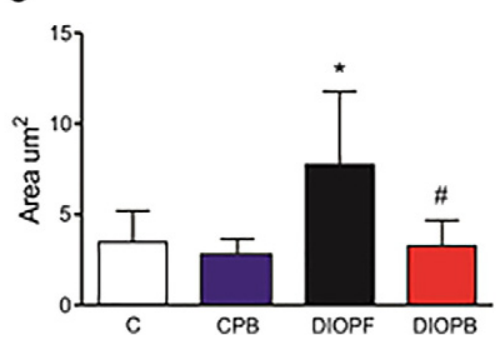

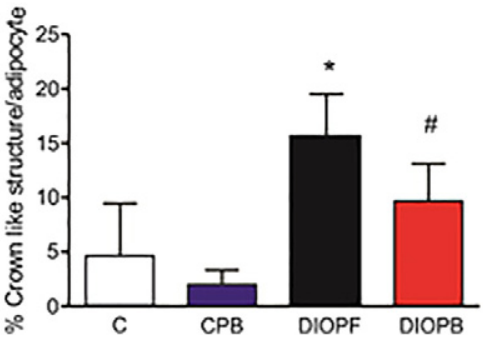

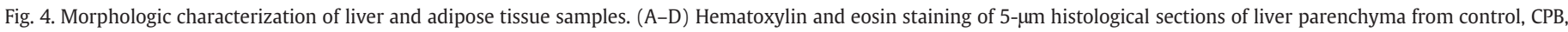

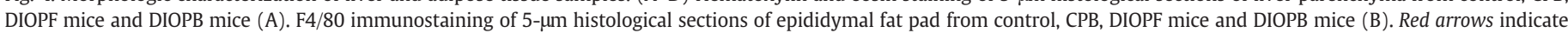

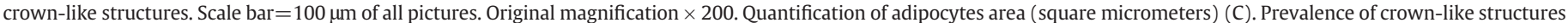
(D). Each bar represents the mean \pm S.E.M. from five different experiments. ${ }^{*} P<.05$ vs. control group and $\# P<.05$ vs. DIOPF. 


\subsection{Effects of probiotics on hypothalamic insulin and leptin signaling}

As mentioned before, probiotic-treated mice receiving an HFD gained less weight than DIO mice (DIOPB $11.4 \pm 1.2 \mathrm{~g} v \mathrm{v}$. DIO $16.9 \pm 1.4 \mathrm{~g}$ during treatment; $P<.05$ ) and had reduced food intake compared with animals that did not receive probiotics (DIOPB $2.9 \pm 0.2 \mathrm{~g} /$ day $v$ s. DIO 3.4 $\pm 0.3 \mathrm{~g} / \mathrm{da} ; P<.01)$. We then investigated whether probiotics treatment improved the response to anorexigenic hormones such as leptin and insulin in mice on HFD. The administration of probiotics in mice fed HFD improved leptin sensitivity, as evidenced by reduced food intake induced by leptin in DIOPF (Fig. 5A). In accordance, there was an increase in JAK2 and STAT3 phosphorylation in the hypothalamus of DIOPB compared to DIOPF mice (Fig. 5B). In addition to greater leptin sensitivity, we observed an enhanced anorexigenic effect in response to ICV insulin administration in the DIOPB group after 4 and $12 \mathrm{~h}$ (Fig. 5C). In parallel, Akt phosphorylation was increased in response to ICV insulin in the hypothalamus of DIOPB compared to DIOPF mice (Fig. 5D). We further measured mRNA levels of NPY and POMC in the hypothalamus since these neuropeptides have major action regulating food intake. Probiotics treatment reduced NPY mRNA without changes in POMC mRNA levels in the hypothalamus of DIOPB compared to DIOPF mice (Fig. 5E and F). We also evaluated the effect of probiotics on several inflammatory signals in the hypothalamus. Similar to the effects observed in peripheral tissues, HFD-fed animals showed increment in TLR4 and IL-6 expression in the hypothalamus, and probiotic treatment was able to reduce TLR4 and IL-6 mRNA levels in this tissue (Fig. 5G and $H)$. There were no changes in the TNF- $\alpha$ mRNA levels in the hypothalamus of DIOPB compared to DIOPF mice (Fig. 5I).

\section{Discussion}

Here we show that HFD animals treated with probiotics have changes in gut microbiota and the intestinal gene expression of metabolic and immunological molecules that resulted in reductions in circulating LPS, which attenuated TLR4 activation and improved insulin signaling and inflammatory profiles in liver and muscle. In addition, the probiotic treatment improved hypothalamic insulin and leptin signaling pathways and modulated the control food intake (Fig. 6).

Our metagenomic analysis of gut microbiota in the DIO groups showed an increase in Bacteroidetes prevalence and a slight reduction in Firmicutes. This result is similar to previous studies [46-48], including a work from our group using also Swiss mice [7], but is not in agreement with other classical published data [8,49], suggesting that the gut microbiota profile in obesity depends also on genetic background. Differences in animal species and/or strain may explain these controversial results. Another phylum that is important to intestinal homeostasis and was reduced in DIOPF animals compared to controls was the Actinobacteria. Mice subjected to probiotic treatment and fed HFD showed some differences in gut microbiota profiles compared with DIO nontreated animals. Despite the maintenance of Bacteroidetes phylum, there were a significant reduction in Firmicutes prevalence and an increase in Actinobacteria. Genera analysis of Bacteroides showed relevant modifications after PB treatment: the genus Bacteroides increased considerably, and it is known that it has beneficial activities to the host, including increased adipose tissue lipolysis, intestinal production of GLP-1, as well as reduction of
A

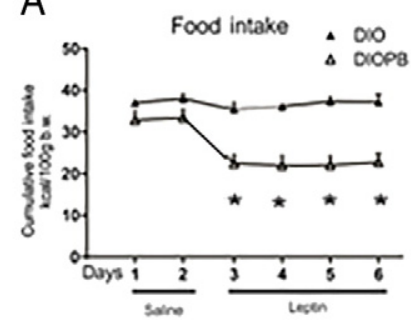

D

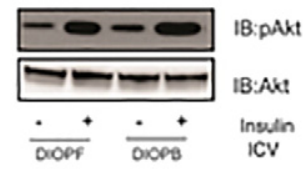

G

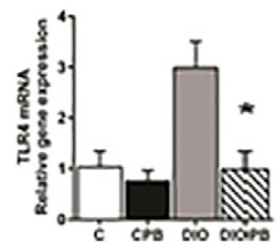

B

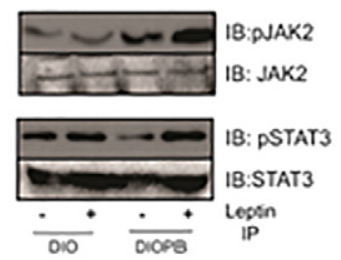

E

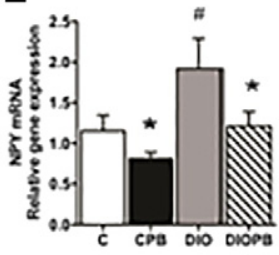

$\mathrm{H}$

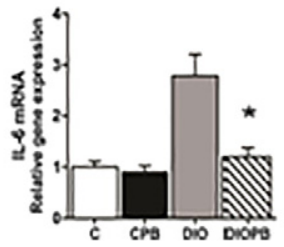

C

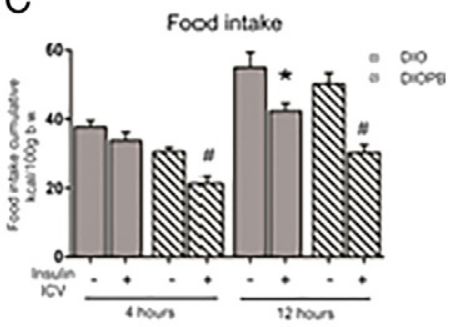

$\mathrm{F}$

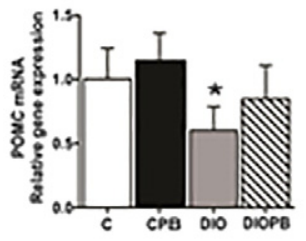

।

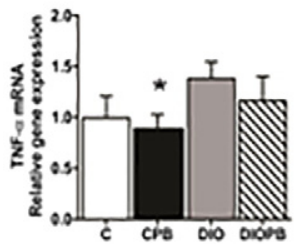

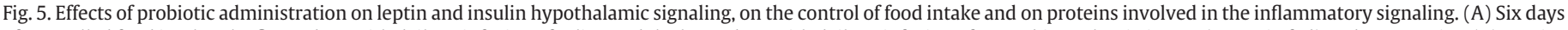

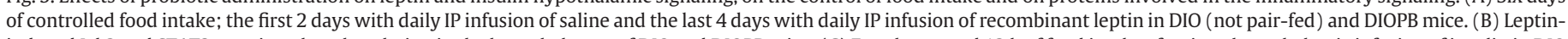

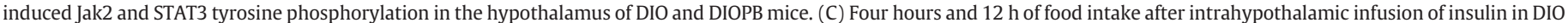

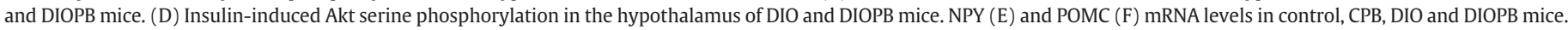

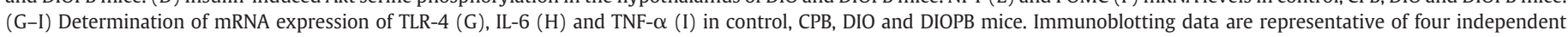

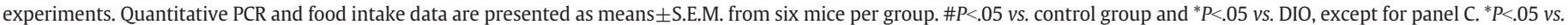
DIO negative, $\# P<.05$ vs. DIOPB negative. IB, immunoblotting; p, phosphorylated. 

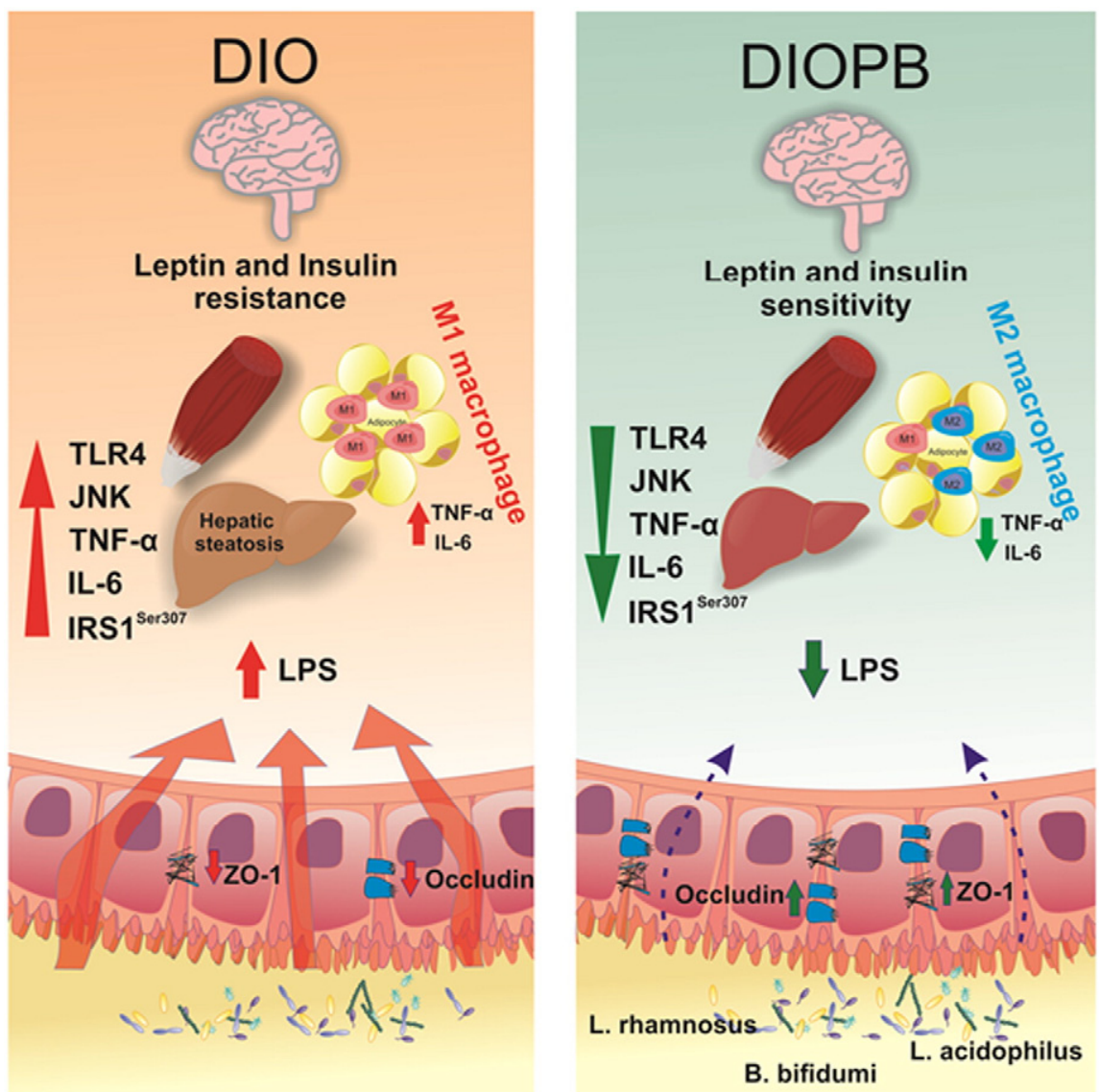

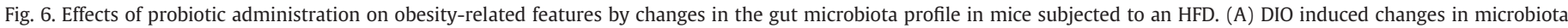

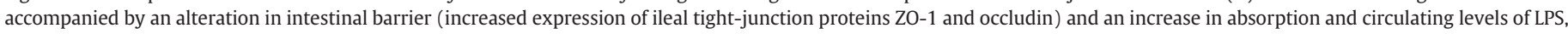

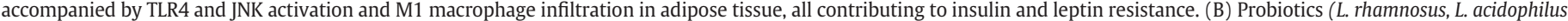
and B. bifidum) treatment by modulating microbiota reversed these inflammatory phenomena and improved insulin and leptin action.

intestinal inflammation [50,51]. Another genus that increased with probiotic administration in DIO animals was Alistipes. Serino et al. [52] described that mice that have high prevalence of this genus is protected from type 2 diabetes in an HFD scenario. Thus, despite the unchanged prevalence of the Bacteroidetes phylum following probiotic treatment, the modulation of the genera of this phylum might have a role in the improvement of glucose tolerance and insulin sensitivity.

Changes in the gut microbiota of DIO mice promoted by probiotics were accompanied by increased expression of ileal tight-junction proteins (ZO-1 and occludin) and proglucagon mRNA, as well as reduced intestinal expression of pattern recognition receptors (PRRs) CD-14 and NOD1. These modulations might play a role in the reduction in circulating levels of LPS and the increase in GLP-1 serum levels. In agreement with our results, Gomes et al. [18] have recently shown that bacterial translocation needs CD-14 and NOD1 PRRs activation in DIO mice; however, probiotic treatment reversed this situation. It is also known that fermented products of probiotics bacteria can increase gut proglucagon expression, which results in GLP-1 and GLP-2 secretion [53,54]. Another important intestinal protein modulated by probiotics is FIAF. Obesogenic microbiota decreases the expression of this molecule, a factor able to increase lipoprotein-lipase-dependent triglyceride storage in adipose tissue and to increase adipocyte triacylglycerol accumulation [5,55]. Our results showed that probiotic administration increased the ileal expression of FIAF in obese mice, probably contributing to reduce fat storage in these animals.
It is interesting that obese mice treated with probiotics showed a reduction in portal LPS concentrations, which certainly contributed to down-regulate the TLR4-MyD88 interaction in liver and muscle, leading to reduced activation of JNK and IRS-1 serine phosphorylation. As expected, in parallel, there was an increase in activation of insulin signaling in liver and muscle, improving insulin sensitivity and glucose tolerance [56,57].

The positive feedback loop between JNK and proinflammatory cytokines can perpetuate a vicious cycle of low-grade inflammatory signaling, contributing to enhanced insulin resistance $[9,58]$. Our findings indicate that the probiotic treatment reduced not only the JNK activation but the TNF $\alpha$, IL- 6 and IL-1 $\beta$ mRNA levels in liver and muscle tissue, probably by reducing $\mathrm{NF \kappa B}$ translocation into the nucleus.

In obesity, adipose tissue macrophages are an important source of cytokine tissue expression [59]. In addition, a reduction in macrophage infiltration can decrease local inflammation in adipose tissue [60]. In accordance, our findings revealed a reduction in macrophage infiltration in the adipose tissue of DIO mice treated with probiotics, as observed by crown-like structures number. These morphological changes, coupled with reduced expression of inflammatory molecules, are factors that may contribute to improve insulin sensitivity in treated animals. Lipid accumulation in the liver is a hallmark of highfat-diet-induced insulin resistance [61]. Although in hepatic sections of DIOPF mice there were a large number of fat vesicles amply distributed across the parenchyma, mice treated with probiotics 
presented liver parenchyma very similar to that of the controls, with no evidence of hepatic steatosis, in parallel to a marked reduction in inflammatory pathways and cytokine expression in this tissue.

Our data showed that animals fed HFD and treated with probiotics presented decreased weight gain and food intake compared to DIO counterparts. The regulation of food intake by the hypothalamus has been extensively investigated and involves nutrients, hormones and neural signals [62]. Leptin and insulin signaling and action in the hypothalamus have profound anorexigenic effects [62]. Hypothalamic insulin signaling promotes transcriptional and electrical events in neurons and activates PI3K/Akt pathway controlling neuropeptides responsible to maintain energy homeostasis. Leptin in the hypothalamus exerts its effects through LR/JAK2/STAT3 pathway also regulating neuropeptide transcription. Both hormones may increase POMC, which is anorexigenic, and decrease the orexigenic NPY mRNA levels $[62,63]$. Obesity state is associated with insulin and leptin resistance in the hypothalamus [64]. The mechanism responsible for the resistance is likely due to, at least in part, aberrant hypothalamic activation of proinflammatory molecules, including TLR4, JNK and IKK $[55,65]$. Here we reported that probiotic administration by gavage in HFD-fed mice induced a striking reduction in hypothalamic TLR4 and IL-6 mRNA levels and a decrease in protein expression of serine kinases JNK and IKK. The reduction of proinflammatory molecules was associated with an improvement of insulin and leptin signaling, action in the hypothalamus and decrease in NPY mRNA levels. These results might contribute to reduce food intake and adiposity in mice treated with probiotics.

Serum GLP1 levels were higher in mice treated with probiotics than their controls. It is well established that activation of GLP1 receptor in multiple sites of the brain alters energy balance [66]. Thus, we cannot rule out a possible contribution of GLP1 lowering body weight and food intake in mice treated with probiotics.

In summary, our data demonstrate that HFD promotes alterations in gut microflora that are reflected in increased intestinal permeability, LPS translocation and systemic low-grade inflammation, resulting in decreased glucose tolerance and hyperphagic behavior. These obesity-related features were reversed by changes in the gut microbiota profile induced by probiotics in mice subjected to an HFD (Fig. 6). In this context, we believe that probiotics can be an important tool to prevent and treat patients with obesity and insulin resistance, but more studies are needed, especially to develop more specific probiotic strains for treating metabolic disorders such as obesity.

Supplementary data to this article can be found online at http://dx. doi.org/10.1016/j.jnutbio.2017.08.006.

\section{Authors' contributions}

R.A.B.: researched data, contributed to discussion, wrote/ reviewed/edited manuscript; N.T.: researched data; A.G.O.: researched data, wrote/reviewed/edited manuscript; B.M.C.: researched data; G.Z.R.: researched data; T.G.A.: wrote/reviewed/ edited manuscript; J.F.V.: researched data; D.G.: researched data; P.O.P.: reviewed/edited manuscript; A.S.: contributed to discussion, wrote/reviewed/edited manuscript; S.T.O.S., M.J.A.S.: contributed to discussion, wrote/reviewed/edited manuscript.

\section{Conflict of interest}

None.

\section{Acknowledgments}

We also acknowledge the financial support from State University of Campinas (FAEPEX), CEPID 201307607-8, OCRC (Centro de Pesquisa em Obesidade e Comorbidades), and INCT (Instituto Nacional de
Ciência e Tecnologia de Obesidade e Diabetes) 573856/2008-7 and from FAPESP (Fundação de Amparo à Pesquisa do Estado de São Paulo) 2012/15009-0, 2016/07122-2 and CAPES/CNPq (Conselho Nacional de Desenvolvimento Científico e Tecnológico). The authors thank $\mathrm{L}$. Janieri (Department of Internal Medicine, UNICAMP, Campinas, São Paulo) and J. Pinheiro (Department of Internal Medicine, UNICAMP, Campinas, São Paulo) for their technical assistance.

\section{References}

[1] Kahn SE, Hull RL, Utzschneider KM. Mechanisms linking obesity to insulin resistance and type 2 diabetes. Nature 2006;444(7121):840-6.

[2] Gregor MF, Hotamisligil GS. Inflammatory mechanisms in obesity. Annu Rev Immunol 2011;29:415-45.

[3] Saad MJ, Santos A, Prada PO. Linking gut microbiota and inflammation to obesity and insulin resistance. Physiology (Bethesda) 2016;31(4):283-93.

[4] Amar J, Chabo C, Waget A, Klopp P, Vachoux C, Bermudez-Humaran LG, et al. Intestinal mucosal adherence and translocation of commensal bacteria at the early onset of type 2 diabetes: molecular mechanisms and probiotic treatment. EMBO Mol Med 2011;3(9):559-72.

[5] Aronsson L, Huang Y, Parini P, Korach-Andre M, Hakansson J, Gustafsson JA, et al. Decreased fat storage by Lactobacillus paracasei is associated with increased levels of angiopoietin-like 4 protein (ANGPTL4). PLoS One 2010;5(9):1-7.

[6] Cani PD, Amar J, Iglesias MA, Poggi M, Knauf C, Bastelica D, et al. Metabolic endotoxemia initiates obesity and insulin resistance. Diabetes 2007;56(7): 1761-72.

[7] Carvalho BM, Guadagnini D, Tsukumo DM, Schenka AA, Latuf-Filho P, Vassallo J, et al. Modulation of gut microbiota by antibiotics improves insulin signalling in high-fat fed mice. Diabetologia 2012;55(10):2823-34.

[8] Turnbaugh PJ, Ley RE, Mahowald MA, Magrini V, Mardis ER, Gordon JI. An obesityassociated gut microbiome with increased capacity for energy harvest. Nature 2006;444(7122):1027-31.

[9] Velloso LA, Folli F, Saad MJ. TLR4 at the crossroads of nutrients, gut microbiota, and metabolic inflammation. Endocr Rev 2015;36(3):245-71.

[10] Caricilli AM, Saad MJ. Gut microbiota composition and its effects on obesity and insulin resistance. Curr Opin Clin Nutr Metab Care 2014;17(4):312-8.

[11] Qin J, Li R, Raes J, Arumugam M, Burgdorf KS, Manichanh C, et al. A human gut microbial gene catalogue established by metagenomic sequencing. Nature 2010; 464(7285):59-65.

[12] Tremaroli V, Backhed F. Functional interactions between the gut microbiota and host metabolism. Nature 2012;489(7415):242-9.

[13] Duca FA, Lam TK. Gut microbiota, nutrient sensing and energy balance. Diabetes Obes Metab 2014;16(Suppl. 1):68-76.

[14] Chow J, Lee SM, Shen Y, Khosravi A, Mazmanian SK. Host-bacterial symbiosis in health and disease. Adv Immunol 2010;107:243-74.

[15] Backhed F, Ding H, Wang T, Hooper LV, Koh GY, Nagy A, et al. The gut microbiota as an environmental factor that regulates fat storage. Proc Natl Acad Sci U S A 2004;101(44):15718-23.

[16] Cani PD, Bibiloni R, Knauf C, Waget A, Neyrinck AM, Delzenne NM, et al. Changes in gut microbiota control metabolic endotoxemia-induced inflammation in highfat diet-induced obesity and diabetes in mice. Diabetes 2008;57(6):1470-81.

[17] Cani PD, Neyrinck AM, Fava F, Knauf C, Burcelin RG, Tuohy KM, et al. Selective increases of bifidobacteria in gut microflora improve high-fat-diet-induced diabetes in mice through a mechanism associated with endotoxaemia. Diabetologia 2007;50(11):2374-83.

[18] Gomes AC, Bueno AA, de Souza RG, Mota JF. Gut microbiota, probiotics and diabetes. Nutr J 2014;13:1-13.

[19] Kootte RS, Vrieze A, Holleman F, Dallinga-Thie GM, Zoetendal EG, de Vos WM, et al. The therapeutic potential of manipulating gut microbiota in obesity and type 2 diabetes mellitus. Diabetes Obes Metab 2012;14(2):112-20.

[20] Laitinen K, Poussa T, Isolauri E. Probiotics and dietary counselling contribute to glucose regulation during and after pregnancy: a randomised controlled trial. $\mathrm{Br} \mathrm{J}$ Nutr 2009;101(11):1679-87.

[21] Chapman CM, Gibson GR, Rowland I. Health benefits of probiotics: are mixtures more effective than single strains? Eur J Nutr 2011;50(1):1-17.

[22] MacPherson CW, Shastri P, Mathieu O, Tompkins TA, Burguière P. Genome-wide immune modulation of TLR3-mediated inflammation in intestinal epithelial cells differs between single and multi-strain probiotic combination. PLoS One 2017; 12(1):e0169847.

[23] Ashraf R, Shah NP. Immune system stimulation by probiotic microorganisms. Crit Rev Food Sci Nutr 2014;54(7):938-56.

[24] Kumar A, Vlasova AN, Liu Z, Chattha KS, Kandasamy S, Esseili M, et al. In vivo gut transcriptome responses to lactobacillus rhamnosus GG and lactobacillus acidophilus in neonatal gnotobiotic piglets. Gut Microbes 2014:5(2):152-64.

[25] Fu YR, Yi ZJ, Pei JL, Guan S. Effects of Bifidobacterium bifidum on adaptive immune senescence in aging mice. Microbiol Immunol 2010;54(10):578-83.

[26] Hong M, Kim SW, Han SH, Kim DJ, Suk KT, Kim YS, et al. Probiotics (Lactobacillus rhamnosus R0011 and acidophilus R0052) reduce the expression of toll-like receptor 4 in mice with alcoholic liver disease. PLoS One 2015;10(2):e0117451. 
[27] Ritze Y, Bárdos G, Claus A, Ehrmann V, Bergheim I, Schwiertz A, et al. Lactobacillus rhamnosus GG protects against non-alcoholic fatty liver disease in mice. PLoS One 2014;9(1):e80169.

[28] Chen D, Yang Z, Chen X, Huang Y, Yin B, Guo F, et al. Effect of Lactobacillus rhamnosus hsryfm 1301 on the gut microbiota and lipid metabolism in rats fed a high-fat diet. J Microbiol Biotechnol 2015;25(5):687-95.

[29] Bercik P, Park AJ, Sinclair D, Khoshdel A, Lu J, Huang X, et al. The anxiolytic effect of Bifidobacterium longum NCC3001 involves vagal pathways for gut-brain communication. Neurogastroenterol Motil 2011;23(12):1132-9.

[30] Bravo JA, Forsythe P, Chew MV, Escaravage E, Savignac HM, Dinan TG, et al. Ingestion of lactobacillus strain regulates emotional behavior and central GABA receptor expression in a mouse via the vagus nerve. Proc Natl Acad Sci U S A 2011; 108(38):16050-5.

[31] Bonaz BL, Bernstein CN. Brain-gut interactions in inflammatory bowel disease. Gastroenterology 2013;144(1):36-49.

[32] Smith CJ, Emge JR, Berzins K, Lung L, Khamishon R, Shah P, et al. Probiotics normalize the gut-brain-microbiota axis in immunodeficient mice. Am J Physiol Gastrointest Liver Physiol 2014:G793-802.

[33] Schele E, Grahnemo L, Anesten F, Hallen A, Backhed F, Jansson JO. The gut microbiota reduces leptin sensitivity and the expression of the obesitysuppressing neuropeptides proglucagon $(\mathrm{Gcg})$ and brain-derived neurotrophic factor (Bdnf) in the central nervous system. Endocrinology 2013;154(10): 3643-51.

[34] Cani PD, Joly E, Horsmans Y, Delzenne NM. Oligofructose promotes satiety in healthy human: a pilot study. Eur J Clin Nutr 2006;60(5):567-72.

[35] Cani PD, Knauf C. How gut microbes talk to organs: the role of endocrine and nervous routes. Mol Metab 2016;5(9):743-52.

[36] Carvalho-Filho MA, Carvalho BM, Oliveira AG, Guadagnini D, Ueno M, Dias MM, et al. Double-stranded RNA-activated protein kinase is a key modulator of insulin sensitivity in physiological conditions and in obesity in mice. Endocrinology 2012; 153(11):5261-74

[37] Handschin C, Chin S, Li P, Liu F, Maratos-Flier E, Lebrasseur NK, et al. Skeletal muscle fiber-type switching, exercise intolerance, and myopathy in PGC-1alpha muscle-specific knock-out animals. J Biol Chem 2007;282(41):30014-21.

[38] Chakravorty S, Helb D, Burday M, Connell N, Alland D. A detailed analysis of $16 \mathrm{~S}$ ribosomal RNA gene segments for the diagnosis of pathogenic bacteria.J Microbiol Methods 2007;69(2):330-9.

[39] Cintra DE, Pauli JR, Araujo EP, Moraes JC, de Souza CT, Milanski M, et al. Interleukin-10 is a protective factor against diet-induced insulin resistance in liver. J Hepatol 2008;48(4):628-37.

[40] Quaresma PG, Reencober N, Zanotto TM, Santos AC, Weissmann L, de Matos AH, et al. Pioglitazone treatment increases food intake and decreases energy expenditure partially via hypothalamic adiponectin/adipoR1/AMPK pathway. Int J Obes (Lond) 2016;40(1):138-46.

[41] Martin TL, Alquier T, Asakura K, Furukawa N, Preitner F, Kahn BB. Diet-induced obesity alters AMP kinase activity in hypothalamus and skeletal muscle. J Biol Chem 2006;281(28):18933-41.

[42] Cho I, Yamanishi S, Cox L, Methe BA, Zavadil J, Li K, et al. Antibiotics in early life alter the murine colonic microbiome and adiposity. Nature 2012;488(7413): 621-6.

[43] Kameyama K, Itoh K. Intestinal colonization by a Lachnospiraceae bacterium contributes to the development of diabetes in obese mice. Microbes Environ 2014; 29(4):427-30.

[44] Qin J, Li Y, Cai Z, Li S, Zhu J, Zhang F, et al. A metagenome-wide association study of gut microbiota in type 2 diabetes. Nature 2012;490(7418):55-60.

[45] Musso G, Gambino R, Cassader M. Obesity, diabetes, and gut microbiota: the hygiene hypothesis expanded? Diabetes Care 2010;33(10):2277-84
[46] Collado MC, Isolauri E, Laitinen K, Salminen S. Distinct composition of gut microbiota during pregnancy in overweight and normal-weight women. Am J Clin Nutr 2008;88(4):894-9.

[47] Schwiertz A, Taras D, Schafer K, Beijer S, Bos NA, Donus C, et al. Microbiota and SCFA in lean and overweight healthy subjects. Obesity (Silver Spring) 2010;18(1): $190-5$.

[48] Henao-Mejia J, Elinav E, Jin C, Hao L, Mehal WZ, Strowig T, et al. Inflammasomemediated dysbiosis regulates progression of NAFLD and obesity. Nature 2012; 482(7384):179-85.

[49] Ley RE, Backhed F, Turnbaugh P, Lozupone CA, Knight RD, Gordon JI. Obesity alters gut microbial ecology. Proc Natl Acad Sci U S A 2005;102(31):11070-5.

[50] Round JL, Mazmanian SK. The gut microbiota shapes intestinal immune responses during health and disease. Nat Rev Immunol 2009;9(5):313-23.

[51] Martinez I, Wallace G, Zhang C, Legge R, Benson AK, Carr TP, et al. Diet-induced metabolic improvements in a hamster model of hypercholesterolemia are strongly linked to alterations of the gut microbiota. Appl Environ Microbio 2009;75(12):4175-84.

[52] Serino M, Luche E, Gres S, Baylac A, Berge M, Cenac C, et al. Metabolic adaptation to a high-fat diet is associated with a change in the gut microbiota. Gut 2012;61(4):543-53.

[53] Le Poul E, Loison C, Struyf S, Springael JY, Lannoy V, Decobecq ME, et al. Functional characterization of human receptors for short chain fatty acids and their role in polymorphonuclear cell activation. J Biol Chem 2003;278(28):25481-9.

[54] Holst JJ. Glucagon and glucagon-like peptides 1 and 2. Results Probl Cell Differ 2010;50:121-35

[55] Carvalho FA, Aitken JD, Vijay-Kumar M, Gewirtz AT. Toll-like receptor-gut microbiota interactions: perturb at your own risk! Annu Rev Physiol 2012;74: 177-98.

[56] Paz K, Hemi R, LeRoith D, Karasik A, Elhanany E, Kanety H, et al. A molecular basis for insulin resistance. Elevated serine/threonine phosphorylation of IRS-1 and IRS-2 inhibits their binding to the juxtamembrane region of the insulin receptor and impairs their ability to undergo insulin-induced tyrosine phosphorylation. J Biol Chem 1997;272(47):29911-8.

[57] Hotamisligil GS, Peraldi P, Budavari A, Ellis R, White MF, Spiegelman BM. IRS-1 mediated inhibition of insulin receptor tyrosine kinase activity in TNF-alpha- and obesity-induced insulin resistance. Science 1996;271(5249):665-8.

[58] Shoelson SE, Lee J, Goldfine AB. Inflammation and insulin resistance. J Clin Invest 2006;116(7):1793-801.

[59] Weisberg SP, McCann D, Desai M, Rosenbaum M, Leibel RL, Ferrante Jr AW. Obesity is associated with macrophage accumulation in adipose tissue. J Clin Invest 2003;112(12):1796-808.

[60] Furuhashi M, Tuncman G, Gorgun CZ, Makowski L, Atsumi G, Vaillancourt E, et al. Treatment of diabetes and atherosclerosis by inhibiting fatty-acid-binding protein aP2. Nature 2007;447(7147):959-65.

[61] Tilg H, Hotamisligil GS. Nonalcoholic fatty liver disease: cytokine-adipokine interplay and regulation of insulin resistance. Gastroenterology 2006;131(3): 934-45.

[62] Morton GJ, Meek TH, Schwartz MW. Neurobiology of food intake in health and disease. Nat Rev Neurosci 2014;15(6):367-78.

[63] Belgardt BF, Bruning JC. CNS leptin and insulin action in the control of energy homeostasis. Ann N Y Acad Sci 2010;1212:97-113.

[64] Myers Jr MG, Leibel RL, Seeley RJ, Schwartz MW. Obesity and leptin resistance: distinguishing cause from effect. Trends Endocrinol Metab 2010;21(11):643-51.

[65] Shi H, Kokoeva MV, Inouye K, Tzameli I, Yin H, Flier JS. TLR4 links innate immunity and fatty acid-induced insulin resistance. J Clin Invest 2006;116(11):3015-25.

[66] Lockie SH. Glucagon-like peptide-1 receptor in the brain: role in neuroendocrine control of energy metabolism and treatment target for obesity. J Neuroendocrinol 2013;25(7):597-604 